\title{
Deeply virtual Compton scattering from Gauge/Gravity duality
}

\section{Miguel S. Costa}

University of Porto

E-mail: miguelc@fc.up.pt

\section{Marko Djuric*}

University of Porto

E-mail: djuric@fc.up.pt

\begin{abstract}
We use gauge/gravity duality to study deeply virtual Compton scattering (DVCS) in the limit of high center of mass energy at fixed momentum transfer, corresponding to the limit of low Bjorken $\mathrm{x}$, where the process is dominated by the exchange of the pomeron. Using conformal Regge theory we review the form of the amplitude for pomeron exchange, both at strong and weak 't Hooft coupling. At strong coupling, the pomeron is described as the graviton Regge trajectory in AdS space, with a hard wall to mimic confinement effects. This model agrees with HERA data in a large kinematical range. The behavior of the DVCS cross section for very high energies, inside saturation, can be explained by a simple AdS black disk model. In a restricted kinematical window, this model agrees with HERA data as well.
\end{abstract}

Sixth International Conference on Quarks and Nuclear Physics

April 16-20, 2012

Ecole Polytechnique, Palaiseau, Paris

\footnotetext{
* Speaker.
} 


\section{Introduction}

The strong interaction is one of the fundamental interactions of nature. It is tempting to attempt to study it perturbatively, as for example quantum electrodynamics, however as is well known the equation for the running of the coupling constant is

$$
\alpha\left(\mu_{1}\right)=\frac{4 \pi}{b_{0} \ln \left(\mu_{1}^{2} / \Lambda_{Q C D}^{2}\right)}
$$

with, $b_{0}=\frac{11}{3} N-\frac{2}{3} n_{f}$. Since the coupling is running in the opposite way to QED, we can use perturbative QCD when all the energy scales in the problem are high, but once the energy scales are of the order of $\Lambda_{Q C D}$ the perturbative expansion breaks down, and we need other techniques to explain experimental results.

Our goal is precisely to apply strong coupling techniques to study real world QCD results. In particular in this note, we will use the so called $A d S / C F T$ correspondance [1] which relates string theory (with gravity) on $A d S_{5} \times S^{5}$ to $\mathscr{N}=4 S Y M$ (a gauge theory close to QCD), and which can be adapted to allow us the study of the strong interaction at strong coupling. Here we will present a short summary of the main results, necessarily skipping over some subtleties and careful analysis, which are presented in detail in [2].

\section{Pomeron in AdS}

In the Regge limit

$$
s \gg t
$$

the Pomeron is the leading order exchange in all total cross sections, and in $2 \rightarrow 2$ amplitudes with the quantum numbers of the vacuum. It is the sum of an infinite number of states with the quantum numbers of the vacuum, and it leads to an amplitude that as $s \rightarrow \infty$ goes as

$$
A(s, t) \sim s^{\alpha(t)}, \alpha(t)=\alpha(0)+\frac{\alpha^{\prime} t}{2},
$$

where $\alpha(t)$ is the Regge trajectory. The Pomeron has been studied since the 1960's, and in perturbative QCD its propagation is given by the BFKL equation.

Now as mentioned before gauge/gravity duality is a proposed exact equivalence between a wide class of theories, with the prototype example being type IIb string theory on $A d S_{5} \times S^{5}$, and $\mathscr{N}=4 S Y M$ living on the boundary of the $A d S$ space. The duality relates states in string theory to operators in the field theory through the relation

$$
\left\langle e^{\int d^{4} x \phi_{i}(x) \mathscr{O}_{i}(x)}\right\rangle_{C F T}=\mathscr{Z}_{\text {string }}\left[\left.\phi_{i}(x, z)\right|_{z \sim 0}\right]
$$

The duality works in the limit

$$
N_{C} \rightarrow \infty, \lambda=g^{2} N_{C}=R^{4} / \alpha^{\prime 2} \gg 1,
$$

and therefore when the coupling is strong. 
The form of the metric on $A d S$ space that we will use is

$$
d s^{2}=e^{2 A(z)}\left[-d x^{+} d x^{-}+d x_{\perp} d x_{\perp}+d z d z\right]+R^{2} d^{2} \Omega_{5} .
$$

$\mathscr{N}=4 S Y M$ is a conformal theory, and as such different from QCD in that it does not incorporate effects of confinement. There are different possibilities one can use in order to put a theory on a space that is only asymptotically AdS, and instead get a dual theory which is not conformal. One such possibility, the one we will use in this study, is to place a sharp cutoff on the radial AdS coordinate at a point $z_{0} \simeq \Lambda_{Q C D}$, and require $z<z_{0}$. The position of the cutoff sets a scale in the theory and thus breaks conformal invariance.

The question then arises, what is the Pomeron in $A d S$ string theory? This was answered by Brower, Polchinski, Strassler and Tan [3], giving the Pomeron as the Regge trajectory of the graviton. The Pomeron exchange propagator in AdS is given by

$$
\mathscr{K}=\frac{2\left(z z^{\prime}\right)^{2} s}{g_{0}^{2} R^{4}} \chi\left(s, b, z, z^{\prime}\right),
$$

where due to conformal invariance $\chi$ is only a function of two variables $\tau$ and $L$, related to the $\log$ of the center of mass energy and the geodesic distance in $A d S$ space respectively.

$$
\chi(\tau, L)=\left(\cot \left(\frac{\pi \rho}{2}\right)+i\right) g_{0}^{2} e^{(1-\rho) \tau} \frac{L}{\sinh L} \frac{\exp \left(\frac{-L^{2}}{\rho \tau}\right)}{(\rho \tau)^{3 / 2}}
$$

According to the Froissart bound

$$
\sigma_{t o t} \leq \pi c \log ^{2}\left(\frac{s}{s_{0}}\right)
$$

and hence the Pomeron exchange violates this bound. Therefore eventually effects beyond one Pomeron exchange become important. One way to include these effects and keep the theory unitary is to study the eikonal approximation in $A d S$ space [4, 5, 6, 7], giving us an expression for the amplitude

$$
A(s, t)=2 i s \int d^{2} l e^{-i \mathbf{l}_{\perp} \cdot \mathbf{q}_{\perp}} \int d z d \bar{z} P_{13}(z) P_{24}(\bar{z})\left(1-e^{i \chi(s, b, z, \bar{z})}\right) .
$$

We can study different scattering processes by supplying different wave functions for the external states, $P_{13}$ and $P_{24}$. For example, this has already been applied to DIS [8], or using the black disk model $[9,10]$.

\section{Deeply Virtual Compton Scattering}

Deeply Virtual Compton Scattering (DVCS) is the scattering between an off-shell photon and a proton, with the outgoing photon being on-shell [11]. We are interested in calculating the differential and exclusive cross sections

$$
\frac{d \sigma}{d t}\left(x, Q^{2}, t\right)=\frac{|A|^{2}}{16 \pi s^{2}}
$$

and

$$
\sigma\left(x, Q^{2}\right)=\frac{1}{16 \pi s^{2}} \int d t|A(s, t)|^{2}
$$


Here $A$ is the amplitude (2.9). We now just need to supply the wavefunctions of the photon and the proton. For the proton we assume a bound state of size $z_{*}$ for which an approximation in AdS/CFT language is a state localized in the bulk around $z_{*}$, so we will take as $P_{24}(\bar{z})=\delta\left(\bar{z}-z_{\star}\right)$. For the photon we can calculate the wave function by evaluating the Witten diagram for the propagation of a gauge current in $A d S$. The wavefunction we calculate this way is $P_{13}(z)=-C \frac{\pi^{2}}{6} K_{1}(Q z)$, with $C$ an unknown normalization constant.

\section{Models}

In our paper [2] we first apply the black disk model to approximate $\chi$ in the amplitude (2.9), but due to time constraints in these talks we are not able to discuss it. Instead we start by the conformal single pomeron exchange, which consists of expanding the amplitude to first order

$$
1-e^{i \chi} \approx-i \chi=-i\left(\cot \left(\frac{\pi \rho}{2}\right)+i\right) g_{0}^{2} e^{(1-\rho) \tau} \frac{L}{\sinh L} \frac{\exp \left(\frac{-L^{2}}{\rho \tau}\right)}{(\rho \tau)^{3 / 2}}
$$

Our model depends on 3 parameters, $z_{*}$ the position of the proton, $\rho=2-j_{0}=\frac{2}{\sqrt{\lambda}}$ and $C g_{0}^{2}$ the product of the aforementioned normalization and $g_{0}^{2}$ is related to the coupling of the external states to the pomeron.

We also look at the hard-wall model, obtained by placing a cutoff at $z=z_{0}$. This modifies the pomeron propagation kernel. In this analysis we approximate it by

$$
\chi_{h w}(\tau, l, z, \bar{z})=C(\tau, z, \bar{z}) D(\tau, l) \chi_{h w}^{(0)}(\tau, l, z, \bar{z})
$$

where $\chi_{h w}(\tau, t=0, z, \bar{z})=\chi(\tau, 0, z, \bar{z})+\mathscr{F}(\tau, z, \bar{z}) \chi\left(\tau, 0, z, z_{0}^{2} / \bar{z}\right), D$ is an exponential cutoff at large $b$, and $C$ is a normalization function which ensures the $t=0$ result, known exactly, is recovered after integrating all $b . F$ is a function that varies between -1 and 1 and can be seen to represent the relative importance of confinement. For the data here analyzed it will roughly vary between -0.1 and -0.4 .

\section{Data Analysis}

We would next like to apply these model to a data analysis. We will compare our expression to the experiments performed at HERA [12,13]. All the data is at small $x(x<0.013)$, which means it is in the region where pomeron exchange is dominant. We have 52 points for the differential and 44 points for the cross section.

For the conformal pomeron the results we get by fitting the differential cross section to the data, we get

$$
g_{0}^{2}=1.95 \pm 0.85, \quad z_{*}=3.12 \pm 0.160 \mathrm{GeV}^{-1}, \quad \rho=0.667 \pm 0.048 .
$$

corresponding to a $\chi_{\text {d.o.f. }}^{2}=1.33$. By fitting the total exclusive cross section we get

$$
g_{0}^{2}=8.79 \pm 4.17, \quad z_{*}=6.43 \pm 2.67 \mathrm{GeV}^{-1}, \quad \rho=0.816 \pm 0.038 .
$$


with a $\chi_{\text {d.o.f. }}^{2}=1.00$. Note that running the same fit using the eikonal approximation, instead of just keeping single pomeron exchange, does not improve the fits, due to the fact that the size of $\chi$ is small in this kinematical region.

Using the hard-wall pomeron our results for the differential cross section are

$$
\begin{gathered}
g_{0}^{2}=2.46 \pm 0.70, \quad z_{*}=3.35 \pm 0.41 \mathrm{GeV}^{-1}, \quad \rho=0.712 \pm 0.038 \\
z_{0}=4.44 \pm 0.82 \mathrm{GeV}^{-1}
\end{gathered}
$$

corresponding to $\chi_{\text {d.o.f. }}^{2}=0.51$. Fitting the cross section we get

$$
\begin{gathered}
g_{0}^{2}=6.65 \pm 2.30, \quad z_{*}=4.86 \pm 2.87 \mathrm{GeV}^{-1}, \quad \rho=0.811 \pm 0.036 \\
z_{0}=8.14 \pm 2.96 \mathrm{GeV}^{-1} .
\end{gathered}
$$

with a $\chi_{\text {d.o.f. }}^{2}=1.03$. We note that for cross section, the fits for the hard-wall and conformal models are equally good, and for the differential cross section the hard-wall fit is better, because for low $t$ some effects of confinement are still felt. In figure 1 we can see a plot of our result for the hard-wall pomeron.
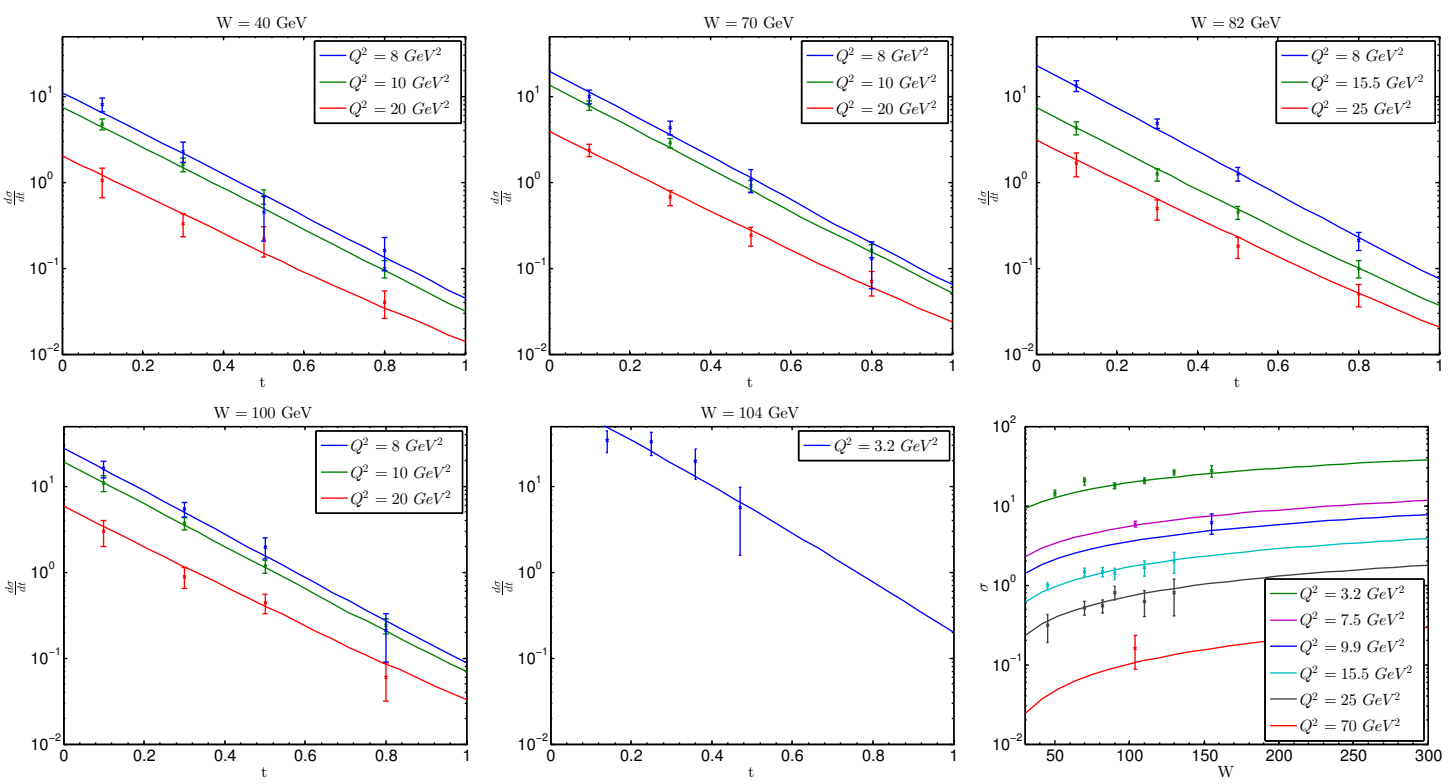

Figure 1: The plots of the hard wall pomeron compared to HERA data. The first 5 correspond to the differential cross section, and the last one to the cross section where we omit some values of $Q^{2}$ to avoid cluttering the graph.

\section{Conclusions}

We have seen that we now have two processes (DIS and DVCS) where the AdS black disk and the AdS (BPST) pomeron exchange give excellent agreement with experiment in the strong coupling region. Hence string theory on AdS is giving us interesting insights into non-perturbative 
scattering. The value of the pomeron intercept is in the region $1.2-1.3$ which is in the crossover region between strong and weak coupling, and a lot of the equations have a form which is very similar both at weak and at strong coupling (but $\chi$ is different). It might therefore be possible to extend some of the insights we gain even into the weak coupling regime. The hard wall model, although a simple modification of AdS, seems to capture effects of confinement well. It will be interesting to repeat some of the calculations using a different confinement model to identify precisely what features are model independent.

We should apply these methods to other processes where pomeron exchange is dominant, eventually having a single set of parameters to describe all pomeron processes. Next step is to study vector meson production, which is similar to DVCS, but with the difference that the outgoing particle is not a photon but a meson. It is also interesting to extend these methods beyond $2 \rightarrow 2$ scattering. A recent paper [14] applies double pomeron exchange to Higgs production, a $2 \rightarrow 3$ scattering process with the exchange of two pomerons.

Acknowledgments The work of M.S.C. and M.D. was partially funded by grants PTDC/FIS/ 099293/2008 and CERN/FP/ 116358/2010. Centro de Física do Porto is partially funded by FCT. The work of M.D. is supported by the FCT/Marie Curie Welcome II program.

\section{References}

[1] J. M. Maldacena, Adv. Theor. Math. Phys. 2 (1998) 231 [hep-th/9711200].

[2] M. S. Costa and M. Djuric, arXiv:1201.1307 [hep-th].

[3] R. C. Brower, J. Polchinski, M. J. Strassler and C. -I Tan, JHEP 0712 (2007) 005 [hep-th/0603115].

[4] R. C. Brower, M. J. Strassler and C. I. Tan, JHEP 0903, 050 (2009)

[5] R. C. Brower, M. J. Strassler and C. I. Tan, JHEP 0903, 092 (2009)

[6] L. Cornalba, M. S. Costa and J. Penedones, JHEP 0806, 048 (2008)

[7] L. Cornalba, M. S. Costa and J. Penedones, JHEP 0709, 037 (2007)

[8] R. C. Brower, M. Djuric, I. Sarcevic and C. I. Tan, JHEP 1011, 051 (2010)

[9] L. Cornalba and M. S. Costa, Phys. Rev. D 78 (2008) 096010

[10] L. Cornalba, M. S. Costa and J. Penedones, Phys. Rev. Lett. 105, 072003 (2010)

[11] K. Kumericki and D. Mueller, Nucl. Phys. B 841 (2010) 1 [arXiv:0904.0458 [hep-ph]].

[12] S. Chekanov et al. [ZEUS Collaboration], JHEP 0905 (2009) 108, arXiv: 0812 . 2517 [hep-ex].

[13] F. D. Aaron et al. [H1 Collaboration], Phys. Lett. B 681 (2009) 391, arXiv: 0907.5289 [hep-ex].

F. D. Aaron et al. [H1 Collaboration], Phys. Lett. B 659 (2008) 796, arXiv: 0709.4114 [hep-ex].

[14] R. C. Brower, M. Djuric and C. -I Tan, arXiv:1202.4953 [hep-ph]. 